\title{
Síntomas, hallazgos radiológicos, endoscópicos y estudios funcionales del esófago en pacientes con hernia hiatal mayor a 5 cm*
}

\author{
Drs. ATTILA CSENDES J. ${ }^{1}$, ITALO BRAGHETTO M. ${ }^{1}$, \\ ANA MARÍA BURGOS L. ${ }^{1}$, T.M. ANA HENRÍQUEZ D. ${ }^{1}$ \\ 1 Departamento de Cirugía, Hospital Clínico de la Universidad de Chile. \\ Santiago, Chile.
}

\begin{abstract}
\section{Clinical and functional features of patients with a hiatal hernia diagnosed during endoscopy}

Background: The finding of a hiatal hernia is usual during upper gastrointestinal endoscopies. However the correlation of symptoms, manometric and radiological findings with the endoscopy report is far from clear. Aim: To assess radiological findings, $24 \mathrm{pH}$ measurements, esophageal manometry and symptoms among patients in whom a hiatal hernia was found during an upper gastrointestinal endoscopy. Material and Methods: One hundred fifteen patients aged 23 to 82 years ( 77 women), with an endoscopic diagnosis of a hiatal hernia of more than $5 \mathrm{~cm}$ were studied. An esophageal manometry and 24 hour $\mathrm{pH}$ determination were carried out. Results: Hiatal hernias type I, II, II and IV were found in 50, 12, 38 and 15 patients of similar age, respectively. Gastroesophageal reflux symptoms were more common among patients with hernias type I and III. Mechanical symptoms such as retrosternal pain were more common in type IV hernias. Manometry showed a hypotensive sphincter in 100 and $78 \%$ of patients with hernias type I and III, respectively. An abnormal 24 hour $\mathrm{pH}$ measurement was found in 95 and $85 \%$ of patients with hernias type I and III, respectively. Conclusions: Functional studies show a high frequency of pathological acid reflux among patients with hiatal hernias type I and III.
\end{abstract}

Key words: Hiatal hernia, esophageal reflux, esophageal manometry.

\section{Resumen}

El hallazgo de una hernia hiatal durante un estudio endoscópico se informa frecuentemente. Sin embargo, no hay referencias específicas acerca de los síntomas y los trastornos funcionales del esófago según el tipo de hernia hiatal. Objetivo: Determinar los síntomas predominantes, los hallazgos radiológicos y endoscópicos $\mathrm{y}$ de los estudios funcionales en pacientes con los diferentes tipos de hernia hiatal. Método: Se evaluaron 115 pacientes, todos con diagnóstico radiológico de hernia hiatal mayor a $5 \mathrm{~cm}$, realizando estudios endoscópicos,

* Recibido el 8 de abril de 2013 y aceptado para publicación el 6 de mayo de 2013.

Los autores no refieren conflictos de interés.

Correspondencia: Dr. Attila Csendes J.

Santos Dumont 999. Santiago, Chile. acsendes@redclinicauchile.cl 
histológicos y manometría esofágica, junto a pHmetría de $24 \mathrm{~h}$. Resultados: La edad fue similar en los 4 tipos de hernia hiatal. Síntomas de reflujo gastroesofágico se presentaron con gran frecuencia en hernias tipo I y III, mientras que síntomas mecánicos se manifestaron en hernias tipo IV. El estudio manométrico mostró un esfínter hipotensivo junto con reflujo ácido patológico en casi $90 \%$ de las hernias tipo I y IIII. Conclusión: El estudio más útil para el diagnóstico de hernia hiatal es el radiológico. La endoscopia es necesaria para comprobar el daño de la mucosa esofágica. Los estudios funcionales muestran una alta prevalencia de reflujo ácido patológico en hernias hiatales tipo I y II.

Palabras clave: Hernia hiatal, endoscopia, pH 24 h, manometría esofágica.

\section{Introducción}

La hernia hiatal es la protrusión de un segmento mayor o menor de estómago a través del orificio hiatal hacia el tórax, acompañado de un saco herniario de origen peritoneal. Estas hernias se clasifican en 4 tipos según el estudio radiológico ${ }^{1,2}$ : tipo I, que corresponde al desplazamiento axial de un segmento gástrico, tipo II, en el que la unión gastroesofágica permanece en el abdomen y se desplaza el fondo gástrico hacia el tórax, tipo III en que existe una combinación del tipo I y II, y tipo IV, en el que además del estómago, se hernian otras estructuras como colon, omento o intestino delgado.

La gran importancia de una hernia hiatal es la frecuente asociación con reflujo gastroesofágico patológico ${ }^{3-6}$. En la literatura quirúrgica internacional y nacional hay una gran cantidad de estudios referentes a hernia hiatal y reflujo gastroesofágico, pero no hemos encontrado una referencia específica que evalúe y compare los síntomas y hallazgos funcionales con los diferentes tipos de hernias hiatales.

El objetivo del presente estudio fue determinar los síntomas predominantes y los hallazgos endoscópicos y de los estudios funcionales del esófago, en pacientes con los diferentes tipos de hernia hiatal.

\section{Material y Método}

\section{Pacientes estudiados}

El grupo comprende a 115 pacientes con hernia hiatal mayor a $5 \mathrm{~cm}$ que fueron atendidos entre enero de 2005 y diciembre de 2011. El criterio de inclusión fue evaluar a pacientes con estudio radiológico que demostrara una hernia hiatal mayor de 5 $\mathrm{cm}$, independiente de sus síntomas. Cada paciente se clasificó según el tipo de hernia descrito previamente en la evaluación radiológica.

Los criterios de exclusión fueron pacientes con hernia hiatal endoscópica menores a $5 \mathrm{~cm}$, esófago de Barrett extenso, adenocarcinoma esófago y esclerodermia del esófago.

\section{Estudio radiológico}

Este examen es el estudio más preciso para determinar la existencia de una hernia hiatal, su tamaño y su tipo ${ }^{3-5}$. Se administró a cada paciente $200 \mathrm{ml}$ de sulfato de bario diluido y se tomaron 10 a 12 placas en diferentes posiciones, según el estándar radiológico. El tamaño de la hernia se midió entre las degluciones, desde el centro de diafragma hasta el aspecto superior de la bolsa gástrica intratorácica, lugar donde terminan los pliegues gástricos. Además se determinó si había algún otro órgano que estaba herniado hacia el tórax.

\section{Estudio endoscópico}

En todos los pacientes se efectuó un completo estudio endoscópico del esófago, estómago y duodeno, empleando el vídeo endoscópico de Olympus ${ }^{\circledR}$ (Tokyo, Japón). Se evaluó el aspecto de la mucosa, lesiones asociadas y en algunos casos se lograron tomar muestras para biopsia a nivel yuxtacardial, inmediatamente por debajo del cambio de mucosas o línea $\mathrm{Z}$.

El tamaño de la hernia se midió determinando la distancia entre el comienzo de los pliegues gástricos y la impresión hiatal durante una respiración tranquila.

\section{Estudio manométrico}

Los detalles de este examen se han publicado anteriormente ${ }^{7,8}$. Los hallazgos más importantes que se consignaron fueron la presión de reposo del esfínter esofágico inferior o gastroesofágico, su largo total y su largo abdominal y la amplitud de las ondas esofágicas en la mitad distal del esófago ${ }^{8}$.

\section{pHmetría de $24 \mathrm{~h}$}

Los detalles de este procedimiento también se han publicado en extenso en estudios previos ${ }^{7,8}$. El resultado final más útil de entre 6 parámetros es el porcentaje total del tiempo en $24 \mathrm{~h}$ en que el $\mathrm{pH}$ intraesofágico, medido $5 \mathrm{~cm}$ por encima del borde proximal del esfinter gastroesofágico, es menor a 4.

\section{Análisis histológico}

En 39 pacientes se tomaron muestras de la unión gastroesofágica distal al cambio de mucosas, que se tiñeron con hematoxilina-eosina y azul de toluidina. Se definió la funditis como presencia de mucosa parietal con células oxínticas y principales junto con 
fenómenos inflamatorios. La carditis corresponde a la presencia de mucosa cardial cilíndrica sin otro tipo de células y con componentes inflamatorios. Metaplasia intestinal corresponde al hallazgo de mucosa intestinal incompleta con células caliciformes, diagnósticos de la presencia de un esófago de Barrett.

\section{Análisis estadístico}

Para la evaluación de la significación estadística, se empleó el test exacto de Fisher y el $\chi^{2}$, tomando un $\mathrm{p}<0,05$ como significativo.

\section{Resultados}

El grupo corresponde a 115 pacientes estudiados, que están dentro del protocolo de análisis prospectivo de pacientes portadores de reflujo gastroesofágico y hernia hiatal, que se ha desarrollado en nuestro centro desde hace 37 años por 2 de los autores (AC y IB).

La Tabla 1 muestra la distribución etaria y por género en los 4 tipos de hernia hiatal. Se aprecia una edad similar en todos los grupos, sin diferencia significativa. En todos los grupos se aprecia un mayor número de mujeres comparado a los hombres, en una proporción de 2:1. En la Tabla 2 se detallan los principales síntomas de cada tipo de hernia hiatal. La suma de porcentaje de síntomas en cada grupo suma más de 100, ya que se consideró cada síntoma por separado. Se aprecia que los pacientes con hernias tipo I y III tienen significativamente más síntomas de reflujo gastroesofágico $(\mathrm{p}<0,001)$, mientras que pacientes con hernia tipo IV tienen otro tipo de síntomas como dolor retroesternal, disnea y cardíacas (taquiarritmia, bloqueo, etc. $(\mathrm{p}<0,00))$. En la Tabla 3 se detalla el tamaño de la hernia según el tipo de hernia evaluado mediante el estudio radiológico. Se aprecia que las hernias tipo I son significativamente más frecuentes entre $5 \mathrm{y}$ $10 \mathrm{~cm}$ de tamaño, comparado con las hernias tipo III $(\mathrm{p}<0,001)$, mientras que las hernias tipo IV son exclusivamente mayores a $15 \mathrm{~cm}$ comparado con los otros grupos $(p<0,01)$. La esofagitis endoscópica se apreció en una proporción similar de casos sin diferencia significativa. Sin embargo, el vólvulo gástrico órgano-axial se presentó exclusivamente en pacientes con hernias tipo III y IV.

En la Tabla 4 se muestra el resultado del análisis histológico de las muestras yuxtacardiales tomadas en 39 pacientes. La presencia de un esófago de Barrett corto se evidenció en una proporción similar de pacientes (entre 1/6 y 1/5 de los casos). Los hallazgos de funditis y carditis fueron también similares.

En la Tabla 5 se señalan los resultados del estudio manométrico en todos los grupos. Los pacientes con hernia tipo I y III tuvieron un importante porcentaje de esfínter gastroesofágico menor a $12 \mathrm{mmHg}$, en contraste con los grupos II y IV y con una presión de reposo promedio significativamente menor que las hernias tipo IV $(\mathrm{p}<0,001)$. El largo abdominal del esfinter en todos fue 0 por la presencia de hernia hiatal. El largo total del esfinter fue significativamente menor en pacientes con tipo I de hernia $(\mathrm{p}<0,001)$. La amplitud de las ondas del esófago distal fue similar en todos los grupos.

En la Tabla 6 se analizó la pHmetría de 24 h en todos los grupos, apreciando que casi la mayoría de los pacientes con hernias tipo I y III presentaron un reflujo gastroesofágico patológico, mientras que en pacientes con hernia tipo II y IV, sólo la mitad de ellos presentaban un reflujo ácido anormal.

Tabla 1. Edad y sexo de pacientes con hernia hiatal mayor de $5 \mathrm{~cm}$ según tipo de hernia. $\mathrm{N}=115$

\begin{tabular}{|lccc|}
\hline & & \multicolumn{2}{c|}{ Sexo } \\
& Edad (años) & Femenino & Masculino \\
Tipo I & $58,5 \pm 12$ & 33 & 17 \\
$\mathrm{n}=50$ & $(23-74)$ & & \\
Tipo II & $60,5 \pm 13$ & 7 & 5 \\
$\mathrm{n}=12$ & $(35-76)$ & & \\
Tipo III & $60,7 \pm 11$ & 27 & 11 \\
$\mathrm{n}=38$ & $(42-77)$ & & 5 \\
Tipo IV & $62,3 \pm 15$ & 10 & 38 \\
$\mathrm{n}=15$ & $(53-82)$ & & \\
Total & & 77 & \\
\hline
\end{tabular}

( ) = rango de edad.

Tabla 2. Síntomas de pacientes con hernia hiatal mayor de $5 \mathrm{~cm}$ según tipo de hernia. $\mathrm{N}=115$

\begin{tabular}{|c|c|c|c|c|}
\hline & \multicolumn{4}{|c|}{ Tipo hernia } \\
\hline & $\begin{array}{c}\mathbf{I} \\
\mathbf{n}=\mathbf{5 0}\end{array}$ & $\begin{array}{c}\text { II } \\
n=12\end{array}$ & $\begin{array}{c}\text { III } \\
\mathbf{n}=\mathbf{3 8}\end{array}$ & $\begin{array}{c}\text { IV } \\
n=15\end{array}$ \\
\hline Pirosis & $45(90)$ & $4(34)$ & $33(87)$ & $5(33)$ \\
\hline Regurgitación & $44(88)$ & $6(50)$ & $34(89)$ & $7(47)$ \\
\hline $\begin{array}{l}\text { Dolor } \\
\text { retroestermal }\end{array}$ & 3 (6) & $1(16)$ & $15(39)$ & $13(87)$ \\
\hline Anemia & 1 (2) & -- & $3(8)$ & $10(67)$ \\
\hline Disnea & -- & 1 (9) & 1 (3) & $14(93)$ \\
\hline Disfagia & -- & -- & $5(13)$ & $1(6,6)$ \\
\hline Cardíacos & -- & -- & -- & $14(93)$ \\
\hline
\end{tabular}

( ) \% pacientes. 
Tabla 3. Hallazgos radiológicos y endoscópicos en pacientes con hernia hiatal mayor de $5 \mathrm{~cm}$ según tipo de hernia. $\mathrm{N}=115$

\begin{tabular}{|lccccc|}
\hline & \multicolumn{4}{c}{ Tipo hernia } \\
& I & II & III & IV \\
& $\mathbf{n = 5 0}$ & $\mathbf{n}=\mathbf{1 2}$ & $\mathbf{n}=\mathbf{3 8}$ & $\mathbf{n}=\mathbf{1 5}$ \\
Tamaño radiológico $(\mathrm{cm})$ & & & & \\
$5-9,9$ & $36(71)$ & $6(45)$ & $9(24)$ & -- \\
$10-14,9$ & $12(25)$ & $2(18)$ & $21(55)$ & -- \\
$>/=15$ & $2(4)$ & $4(37)$ & $8(21)$ & $15(100)$ \\
Esofagitis endoscópica & $22(45)$ & $2(18)$ & $5(13)$ & 5 & $(33)$ \\
Vólvulo gástrico & -- & -- & $7(18)$ & 7 & $(47)$ \\
\hline
\end{tabular}

( ) $\%$ casos.

Tabla 4. Hallazgos histológicos a nivel yuxtacardial en pacientes con hernia hiatal según tipo de hernia. $\mathbf{N}=\mathbf{3 9}$

\begin{tabular}{|lccc|}
\hline & \multicolumn{3}{c|}{ Tipo hernia } \\
& $\mathbf{n = 1 4}$ & $\begin{array}{c}\text { II } \\
\mathbf{n}=\mathbf{7}\end{array}$ & $\begin{array}{c}\text { III } \\
\mathbf{n}=\mathbf{1 8}\end{array}$ \\
Funditis & $5(36)$ & $1(14)$ & $8(44)$ \\
Carditis & $6(43)$ & $1(14)$ & $8(44)$ \\
$\begin{array}{l}\text { Metaplasia intestinal } \\
\text { (Barrett corto) }\end{array}$ & $3(21)$ & $1(14)$ & $3(17)$ \\
\hline
\end{tabular}

( ) $\%$ casos.
En la Figura 1 se comparan los resultados de la presión de reposo promedio en cada grupo con la pHmetría de $24 \mathrm{~h}$ promedio. Se aprecia una clara correlación entre ambos estudios.

\section{Discusión}

Los resultados del presente estudio generan varias interrogantes que comentaremos:

\section{Definición de hernia hiatal}

Aunque este punto parece muy obvio, nos parece que hay un abuso endoscópico de este diagnóstico, y que trae como consecuencia indicación quirúrgica muy discutible. La definición de cualquiera hernia abdominal es la protrusión de contenido intestinal a través de un orificio natural o adquirido y con un saco herniario.

Por lo tanto, la hernia hiatal que nosotros llamamos como "verdadera" debe tener un saco herniario y el hiatus diafragmático dilatado. Por eso que escogimos para el presente estudio hernias hiatales mayores de $5 \mathrm{~cm}$, ya que todos los pacientes incluidos en nuestra investigación, cumplían con los requisitos

Tabla 5. Estudio manométrico en pacientes con hernia hiatal mayor de $5 \mathrm{~cm}$ según tipo de hernia

\begin{tabular}{|c|c|c|c|c|}
\hline & \multicolumn{4}{|c|}{ Tipo hernia } \\
\hline & $\begin{array}{c}I \\
\mathbf{n}=\mathbf{5 0}(\mathrm{A})\end{array}$ & $\begin{array}{c}\text { II } \\
\mathrm{n}=\mathbf{1 2}(\mathrm{B})\end{array}$ & $\begin{array}{c}\text { III } \\
\mathbf{n}=\mathbf{3 8}(\mathrm{C})\end{array}$ & $\begin{array}{c}\text { IV } \\
\mathrm{n}=\mathbf{1 5}(\mathrm{D})\end{array}$ \\
\hline 1. Presión reposo EGE (mmHg) & $8,3 \pm 2,1$ & $11,1 \pm 1,6$ & $9,1 \pm 2,8$ & $16,1 \pm 3,2$ \\
\hline$\%$ casos $<12 \mathrm{mmHg})$ & 100 & 43 & 78 & 46 \\
\hline 2. Largo abdominal (mm) & 0 & 0 & 0 & 0 \\
\hline 3. Largo total (mm) & $23,1 \pm 10$ & $35 \pm 8$ & $28,5 \pm 9$ & $33,2 \pm 10$ \\
\hline 4. Amplitud ondas esófago distal $(\mathrm{mmHg})$ & $48 \pm 15$ & $69,3 \pm 22$ & $81,2 \pm 20$ & $53,2 \pm 15$ \\
\hline
\end{tabular}

Tabla 6. pHmetría de 24 h en pacientes con hernia hiatal mayor de $5 \mathrm{~cm}$ según tipo de hernia

\begin{tabular}{|c|c|c|c|c|}
\hline \multirow[b]{2}{*}{$\%$ tiempo con $\mathrm{pH}>$ a 4 en $24 \mathrm{~h}$} & \multicolumn{4}{|c|}{ Tipo hernia } \\
\hline & $\begin{array}{c}I \\
\mathrm{n}=\mathbf{3 8}\end{array}$ & $\begin{array}{c}\text { II } \\
n=7\end{array}$ & $\begin{array}{c}\text { III } \\
n=33\end{array}$ & $\begin{array}{c}\text { IV } \\
\mathrm{n}=13\end{array}$ \\
\hline Normal < 4\% & 2 & 3 & 5 & 6 \\
\hline promedio & 2,4 & 3,7 & 0,8 & 3,1 \\
\hline Anormal > 4\% & $36(95)$ & $4(57)$ & $28(85)$ & $7(54)$ \\
\hline promedio & $15,2 \pm 8,3$ & $12,2 \pm 4$ & $26,0 \pm 12,5$ & $34,1 \pm 21,5$ \\
\hline
\end{tabular}

( ) $\%$ casos. 


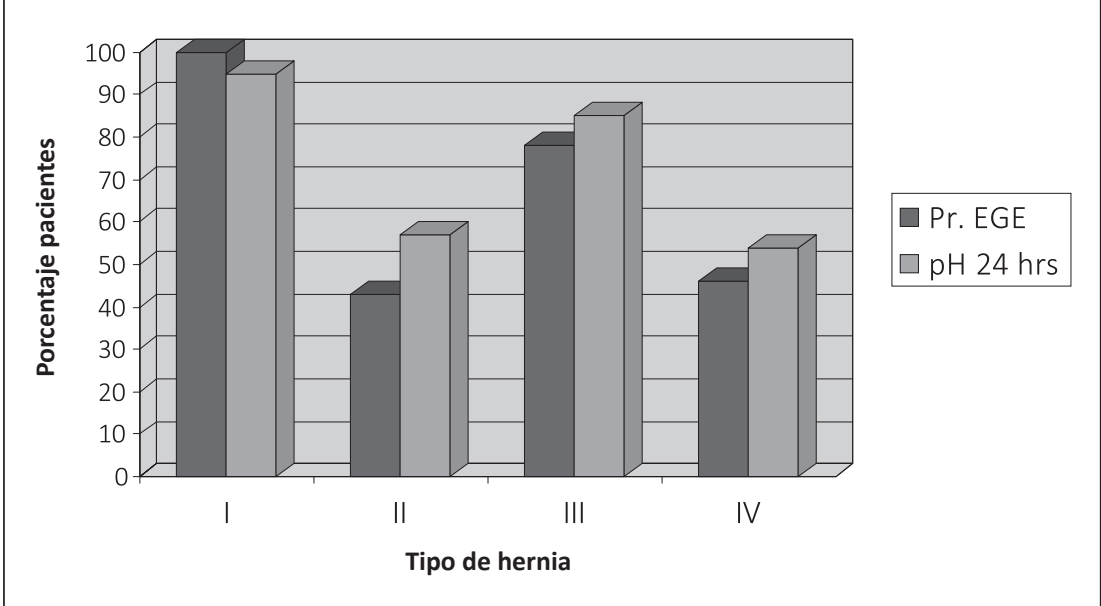

Figura 1. Porcentaje de pacientes con presión de reposo EGE menor a $12 \mathrm{mmHg}$ y $\%$ pacientes con reflujo ácido patológico según tipo de hernia. enunciados. En nuestra extensa experiencia en este tema, los pacientes con el diagnóstico de "hernia hiatal" endoscópica de 2 a $3 \mathrm{~cm}$ nunca corresponden a una hernia hiatal verdadera, con saco herniario, sino que simplemente un ascenso de unos $\mathrm{cm}$ de pliegues gástricos durante una distensión gástrica por el aire introducido con el endoscopio ${ }^{6}$. Estos pacientes cuando se operan por un reflujo ácido patológico, jamás tienen un hiatus muy dilatado y nunca tienen un saco herniario. Por estos motivos insistimos en un correcto diagnóstico de hernia hiatal "verdadera".

\section{Diagnóstico de hernia hiatal}

Clásicamente el diagnóstico de hernia hiatal se basaba en un buen estudio radiológico al demostrar la presencia de una bolsa gástrica o pouch que se extiende desde el centro del diafragma hasta la prolongación proximal de los pliegues gástricos, medidos durante respiración normal. Este criterio es el válido actualmente por todos los investigadores $^{3-8}$. En contraste, el diagnóstico endoscópico es más difícil e impreciso. Durante la endoscopia, la unión gastroesofágica o cardias (donde está el esfínter gastroesofágico) se diagnostica por el lugar donde ocurre la transición del esófago tubular hasta los pliegues gástricos más proximales. Por lo tanto, la correcta identificación de la unión gastroesofágica no es fácil. El diagnóstico endoscópico de hernia hiatal se hace por el desplazamiento de al menos 2 $\mathrm{cm}$ de los pliegues gástricos por sobre el hiatus diafragmático durante una respiración quieta. Por estos motivos, el largo y tamaño preciso de una hernia es difícil y no consistente ${ }^{3,4}$. Lo que es más importante durante el estudio endoscópico es la precisión del aspecto macroscópico de la válvula cardial, según lo propuesto por Hill ${ }^{9,10-12}$, ya que tienen estrecha correlación con la presencia y severidad de reflujo gastroesofágico.

\section{Alteraciones fisiopatológicas de una hernia hiatal}

La presencia de una hernia hiatal altera profundamente la topografía de la unión gastroesofágica reduciendo la presión esfinteriana y al largo de la porción del esfínter gastroesofágico que está debajo del hiatus (esófago abdominal) ${ }^{7,13}$. Desaparece el ángulo de $\mathrm{His}^{2,3}$, alterando la geometría normal del cardias y la tensión de la pared gástrica ${ }^{6}$. Se altera el vaciamiento del esófago hacia el estómago ${ }^{14-16}$, lo que prolonga los episodios de reflujo. Por lo tanto, el reflujo patológico es más probable que ocurra en pacientes con hernia hiatal que en aquellas sin hernia. Además la falla de la competencia esfinteriana es progresiva, dependiendo de la magnitud de la herniación ${ }^{14}$.

\section{Síntomas de una hernia hiatal}

En la literatura internacional hay numerosas publicaciones referentes a síntomas de un paciente con hernia hiatal, mostrando la mayoría una estrecha correlación con síntomas de reflujo gastroesofágico. Sin embargo, no hemos encontrado una descripción clínica de los síntomas según el tipo de hernia diagnosticada al estudio radiológico. Con cierta frecuencia se habla de hernias "encarceladas" o volvulados, sin precisar claramente el tipo de hernia. En una clásica descripción sobre este tema, el grupo de Pearson $^{17}$, reporta síntomas de hernia "masiva" similares a los encontrados en nuestros pacientes con hernias tipo III y IV: vólvulo órgano-axial en 50\%, dolor torácico en $56 \%$, disfagia en $48 \%$ y anemia en $38 \%$. El problema que estos autores describieron una gran frecuencia de "esófago corto" en estos pacientes por mediaciones manométricas, encontrando un 
largo esofágico de $15 \mathrm{~cm}$. Esto contrasta con nuestra experiencia y fue refutada por Cameron ${ }^{18}$ de la Clínica Mayo, quien claramente destaca que el reflujo gastroesofágico no causa acortamiento esofágico, que cuando hay hernia hiatal hay tortuosidad del esófago distal por desplazamiento de la unión GE hacia el tórax y por lo tanto, esófago corto no existe.

\section{Estudios funcionales del esófago}

El presente estudio es el primero que analiza las características funcionales del esófago en pacientes con diferentes tipos de hernia hiatal. Por lo tanto, nuestros resultados son originales e imposibles de comparar. Hay autores que describen un esfínter gastroesofágico en promedio hipotensivo en un 51\% de pacientes con hernia hiatal ${ }^{17}$. La importancia de nuestros hallazgos radica en el hecho que demostramos que pacientes con hernia hiatal tipo I y III se acompañan en una alta frecuencia con síntomas de reflujo GE, mientras que pacientes con hernias tipo II y IV se acompañan de mayor frecuencia con síntomas mecánicos. Nuestros resultados indican una estrecha correlación entre la presencia de un esfínter GE mecánicamente incompetente y presencia de reflujo ácido patológico con la pHmetría de $24 \mathrm{~h}$.

\section{¿Se justifica realizar una fundoplicatura junto con la hernioplastía hiatal?}

Esta pregunta la planteamos en un estudio nuestro previo $^{19}$. A la luz de nuestros estudios endoscópicos $\mathrm{y}$ funcionales de pacientes con hernia hiatal, nos parece altamente recomendable agregar una fundoplicatura cada vez que se realiza una reparación herniaria en pacientes con hernia hiatales mayores a $5 \mathrm{~cm}$. Esto se debe no sólo a la altísima frecuencia de reflujo patológico en pacientes con hernias tipo I y III (que por lejos son las más frecuentes), sino que la profunda alteración anatómica en pacientes con hernias tipo II y IV de la unión gastroesofágica acompañado de reflujo patológico en $50 \%$ de los pacientes, hacen aconsejable agregar una fundoplicatura durante la reparación quirúrgica. Por lo demás no es fácil realizar estudios funcionales en estos pacientes, ya sea por rechazo del paciente o por la imposibilidad de pasar el catéter manométrico hacia el estómago por distorsión anatómica o un vólvulo gástrico.

En resumen, nuestros resultados indican que ante la sospecha de una hernia hiatal, el examen de elección es un estudio radiológico. Este procedimiento nos demuestra si hay o no hernia hiatal, el tipo de hernia y su tamaño. Siempre es conveniente agregar un estudio endoscópico, para comprobar el daño de la mucosa esofágica y la presencia de un esófago de Barrett. Es recomendable realizar estudios funcionales para comprobar la repercusión de la hernia hiatal en la dinámica actividad del esófago. Por último, nos parece que toda hernia hiatal mayor a $5 \mathrm{~cm}$ debiera operarse, ya que los síntomas progresan en el tiempo y en general son pacientes sobre 60 años. Durante la cirugía recomendamos agregar una fundoplicatura junto con la reparación herniaria.

\section{Referencias}

1.- Johnson DA, Ruffin WK. Hiatal hernia. Gastroent Endosc Clin N Am. 1999;6:641-66.

2.- Kaul B, Petersen H, Myrvold HE, Grette K. Hiatus hernia in gastroesophageal reflux disease. Scand J Gastroent. 1986;21:31-4.

3.- Kinoshita Y, Adachi K. Hiatal hernia and gastroesofageal flap value as diagnostic indicators in patients with gastroesofageal reflux disease. J Gastroent. 2006; 41:720-1.

4.- Avidan B, Sonnenberg A, Schall TG, Arefflec G, Metz A, Sontag SJ. Hiatal hernia size, Barrett's length and severity of acid reflux are all risk factors for esophageal adenocarcinoma. Am J Gastroent. 2002;97:1930-6.

5.- Hu AN, Tseng L, Bernstein L. Hiatal hernia, reflux symptoms, body size and risk of esophageal and gastric adenocarcinoma. Cancer 2003;98:940-8.

6.- Savas N, Dagli U, Sahim B. The effect of hiatal hernia on gastroesophageal reflux disease and influence on proximal and distal esophageal reflux. Dig Dis Sci. 2008;53:2380-6.

7.- Csendes A, Burdiles P, Álvarez F, Maluenda F, Henríquez A, Quezada $\mathrm{S}$, et al. Manometric features of mechanically effective lower esophageal sphincter in controls and in patients with different degrees of gastroesophageal reflux. Dis Esoph. 1996;9:290-4.

8.- Csendes A, Álvarez F, Burdiles P, Braghetto I. Magnitud del reflujo ácido gastroesofágico medido por pHmetría de 24 h y manometría comparando el grado de esofagitis endoscópica. Rev Med Chile 1994;122:59-67.

9.- Jones MP, Sloan SS, Rabine JC, Elvert CC, Huang CF, Kahrilas PS. Hiatal hernia is the dominant determinat of esophagitis presence and severity in gastroesophageal reflux disease. Am J Gastroent. 2001;96:1711-7.

10.- Avidan B, Sonnenberg A, Schnell TG, Sontag SJ. Hiatal hernia and acid reflux frequency predict presence and length of Barrett's esophagus. Dig. Dis. Sci. 2002;47:256-264.

11.- Hill AD, Kozarek RA, Kraemer SJM, Aye RW, Mercer D. The gastroesophageal flap value: in vitro and in vivo observation. Gastroent Endosc. 1996;44:541-7.

12.- Falavigna M, Csendes A, Henríquez A, Luengas R. Comparación entre el aspecto endoscópico del cardias, hallazgos manométricos y pHmetría de 24 h en pacientes con síntomas de reflujo gastroesofágico crónico. Rev Med Chile 2006;134:187-92.

13.- Oberg S, Peters JH, DeMeester TR, Lord RV, Johanson 
J. Endoscopic grading of the gastroesophageal value in patients with symptoms of gastroesophageal reflux disease. Surg Endosc. 1999;13:1184-8.

14.- Kahrilas PJ, Lin S, Chen J, Marka M. The effect of hiatus hernia on gastroesophageal junction pressure. Gut 1999;44:476-82.

15.- Sloan S, Rademaker AN, Kabrilas PJ. Determinants of gastroesophageal junction incompetence hiatal hernia, lower esophageal sphincter or both? Ann Interv Med. 1992;117:977-82.

16.- Emerenziani S, Habib FI, Ribolsi M, Cariglia R, Guani- ro MPL, Petitli T. Effect of hiatal hernia esophageal acid clearance in gastro-esophageal reflux disease patients. Aliment Pharmacol Ther. 2006;23:757-4.

17.- Maziak DE, Todd TR, Pearson FG. Massive hiatus hernia: evaluation and surgical management. J Thor Cardiov Surg. 1998;115:53-62.

18.- Cameron AJ. Barrett's esophagus: prevalence and size of hiatal hernia. Am J Gastroent. 1999;94:2054-9.

19.- Braghetto I, Korn O, Csendes A, Burdiles P. Postoperative results after laparoscopic approach for treatment of large hiatal hernias. Inter Surg. 2010;95:80-7. 